\title{
Congenital non-progressive peripheral neuropathy with arthrogryposis multiplex
}

\author{
G. M. YUILL AND P. G. LYNCH \\ From the Department of Neurology, Manchester Royal Infirmary, \\ and the Group Laboratory, Preston Royal Infirmary, Lancashire
}

SYNOPSIS A family is described in which a hereditary peripheral neuropathy occurs, inherited as an autosomal dominant character. The syndrome is present at birth and does not show any significant progression thereafter. Three of the cases have suffered from arthrogryposis multiplex congenita. A secondary myopathy is also present. The condition appears to be intermediary between peroneal muscular atrophy and Friedreich's ataxia. Appearance at birth, presence of arthrogryposis multiplex congenita, and the non-progressive nature separate it sharply from the other heredofamilial ataxias and peripheral neuropathies.

Between clearly defined cases of established syndromes are to be found diverse intermediary examples which do not fit into the existing classifications. Roth (1948) reported two unrelated families in each of which were individuals with clinical signs common to both peroneal muscular atrophy and Friedreich's ataxia. No pathological studies were undertaken. A brief review of the literature suggests that peroneal muscular atrophy and Friedreich's ataxia can be regarded as polar extremes separated by a wide spectrum of slightly differing syndromes. In this report a family is described which seems to fall within this continuum. No similar cases have been recorded, although in some respects it resembles the scapuloperoneal muscular atrophy described by Kaeser (1965).

The essential features of the syndrome are wasting of the feet, calves, peronei, quadriceps, and hamstrings muscles and extensor plantar responses. There are no abnormalities in the trunk or upper limbs. Another most unusual feature is that in all cases some degree of arthrogryposis multiplex congenita was present, which is not seen in the majority of genetically determined nervous diseases.

\section{FAMILY HISTORY}

The proband was a 4 year old boy. His father, sister, and grandfather were also affected. The family was seen in the Department of Neurology at Manchester Royal Infirmary during 1970. In view of his age, investigations on the proband were limited, but they were detailed in the case of his father.

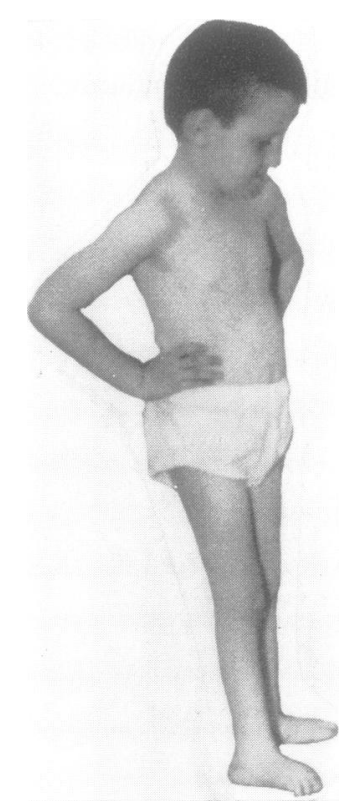

FIG. 1. The proband. Case 1 (J.M. 4-8). 


\section{CASE 1}

J.M. (4-8) was a 4 year old boy (Fig. 1). At birth he was delivered by Caesarean section because of breech presentation and weighed $2.5 \mathrm{~kg}$. He was found to have bilateral talipes equinovalgus. His feet were straightened by serial plasters and manipulation and were in a neutral position at the age of 10 months. His milestones of development were normal. When he was 4 years old his parents became worried by his abnormal gait and sought medical advice. On examination his blood pressure was normal. The heart, lungs, and abdominal viscera were normal. The cranial nerves and nervous system of upper limbs and trunk were normal. There was no ataxia. In

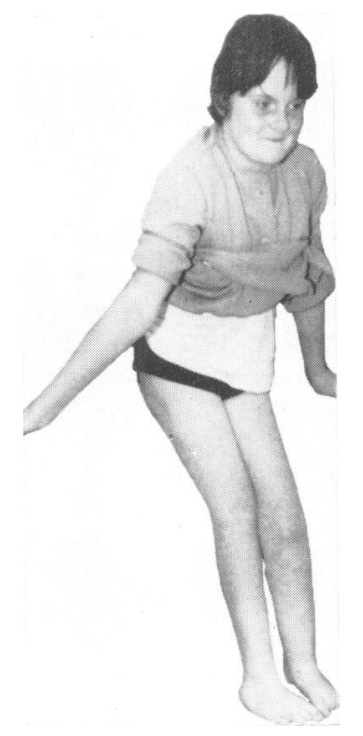

FIG. 2. Case 2 (S.M. 4-9).

the lower limbs there was mild weakness and wasting of the quadriceps and hamstrings and severe weakness and wasting of the anterior tibial, lateral peroneal, and calf muscles. The tone was moderately increased. The abdominal reflexes were present. The knee and ankle jerks were pathologically brisk and the plantar responses were extensor. There was no disturbance of sensation. The gait was spastic. He stood with a marked lumbar lordosis. There was a $10^{\circ}$ flexion deformity of both hips. The feet were normal in size and in a neutral position, but there was considerable limitation of passive dorsiflexion.

The blood haemoglobin level was $14 \mathrm{~g} / 100 \mathrm{ml}$., blood white cell count 7,000/cu.mm, and the ESR (Wintrobe) was $2 \mathrm{~mm}$ in the first hour. Radiographs of the chest were normal. A lumbar myelogram performed under general anaesthesia showed that the anterior-posterior diameter of the cervical canal was of almost adult proportions. No other abnormalities were seen. The cerebrospinal fluid was clear and colourless. It contained 120 red blood cells/ cu.mm, no white blood cells, the protein concentration was $20 \mathrm{mg} / 100 \mathrm{ml}$, and the Lange curve was normal.

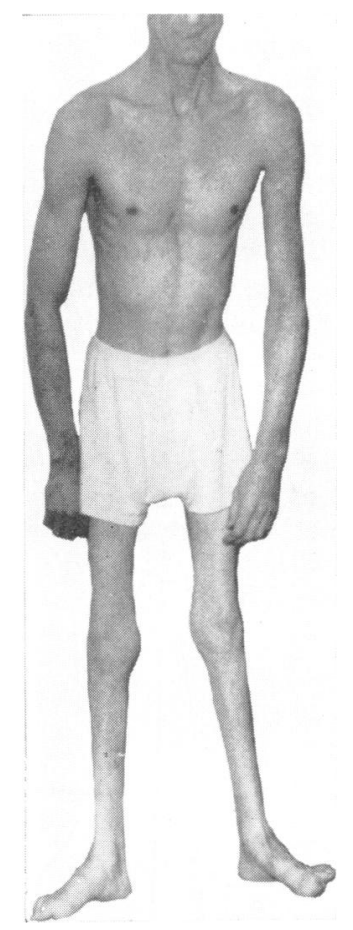

FIG. 3. Case 3 (J.M. 3-8).

CASE 2

S.M. was a 10 year old girl (4-9), sister of the proband (4-8) (Fig. 2). Her obstetric history was normal. After birth her mother noticed that she did not move her legs normally. She had bilateral congenital dislocation of the hips. The hips were deformed, being fixed in $90^{\circ}$ of flexion and $90^{\circ}$ of external rotation. The left foot was in severe equinus and the right one in maximal calcaneovalgus deformity. By the age of 2 years she had had two 
operations to correct the deformity of the left foot, a closed reduction of the left hip joint, and an open reduction of the right hip joint. She was considered to have arthrogryposis multiplex congenita. She never learned to take more than a few steps and went to a school for disabled children. She was first seen in the department at the age of 10 years. Abnormalities were confined to the nervous and locomotor systems. She was of dull normal intelligence. The cranial nerves were normal. There was no ataxia. The upper limbs were normal except for the absence of the reflexes. The trunk was normal. In the lower limbs there was severe weakness and moderate wasting of the quadriceps, anterior tibial, calf, and lateral peroneal musculature. The tone was normal. The abdominal reflexes were absent. The knee and ankle jerks were absent and both plantar reflexes were extensor. Sensation was normal. There was no disturbance of sphincter control. There was a $15^{\circ}$ flexion deformity in both knees. Passive abduction of both hips was greatly restricted. The left foot was in mild calcaneovalgus and the right one was in neutral position. There was severe limitation of passive movements at the ankle and midtarsal joints.

\section{CASE 3}

J.M. (3-8), father of cases 1 and 2, was a 33 year old man who worked as a lathe operator (Fig. 3). His birth was normal. At birth deformities of both feet were noticed and he did not learn to walk until the age of 5 years. He was refused for military service on account of this disability. His only complaints were of a clumsy gait, weakness of the legs, and of chronic metatarsalgia. A wedge tarsectomy was performed on the right foot at the age of 31 years and a wedge fusion of the left midtarsal joint at the age of 32 years. On examination he was a tall man of medium build. Abnormalities were confined to the nervous and locomotor systems. In the nervous system there were no abnormalities in the cranial nerves, upper limbs, or trunk. There was no ataxia. In the lower limbs there was severe wasting and moderate weakness of the quadriceps, hamstring, calf, and peroneal muscles. The tone was normal. The abdominal reflexes were present. The knee jerks were normal but the ankle jerks were depressed. Both plantar responses were extensor. He stood with a mild lumbar lordosis and walked with a waddling gait. Movements of the spine were painless and normal. There was a reduction of passive movement of the ankle and midtarsal joints. The following investigations were performed: blood haemoglobin level $15 \mathrm{~g} /$ $100 \mathrm{ml}$., white blood cell count 8,700 cu.mm ESR (Wintrobe) $2 \mathrm{~mm}$ in the first hour. Serum enzyme activity was: aldolase 2 , creatine phosphokinase 10 , lactic dehydrogenase 275 units. (These are within the normal range for this laboratory). The cerebrospinal fluid (CSF) was clear and colourless and at normalo pressure. There were no cells; the CSF protein was $50 \mathrm{mg} / 100 \mathrm{ml}$., there was no increase in the globulin, and the Lange curve was normal. The WR was negative in both the blood and the cerebrospinato. fluid. Plain radiographs of the chest and lumbar spine?

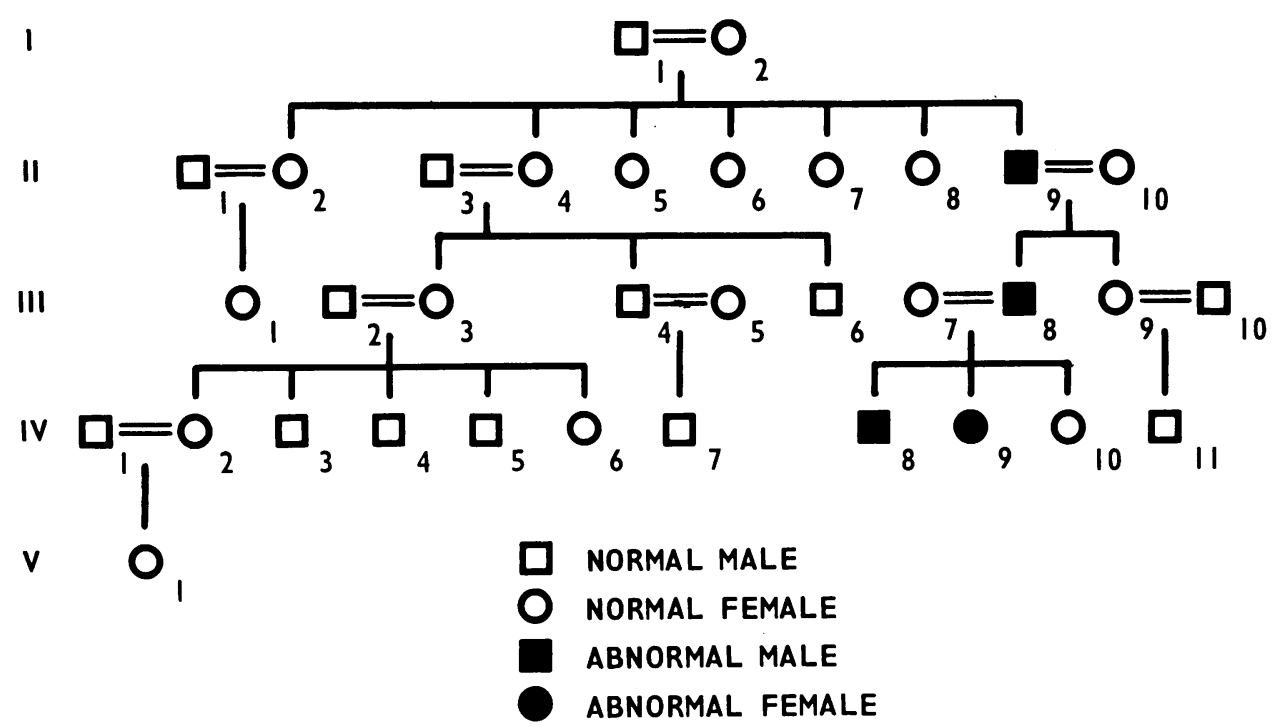

FIG. 4. The family pedigree. For numbering see the text. 


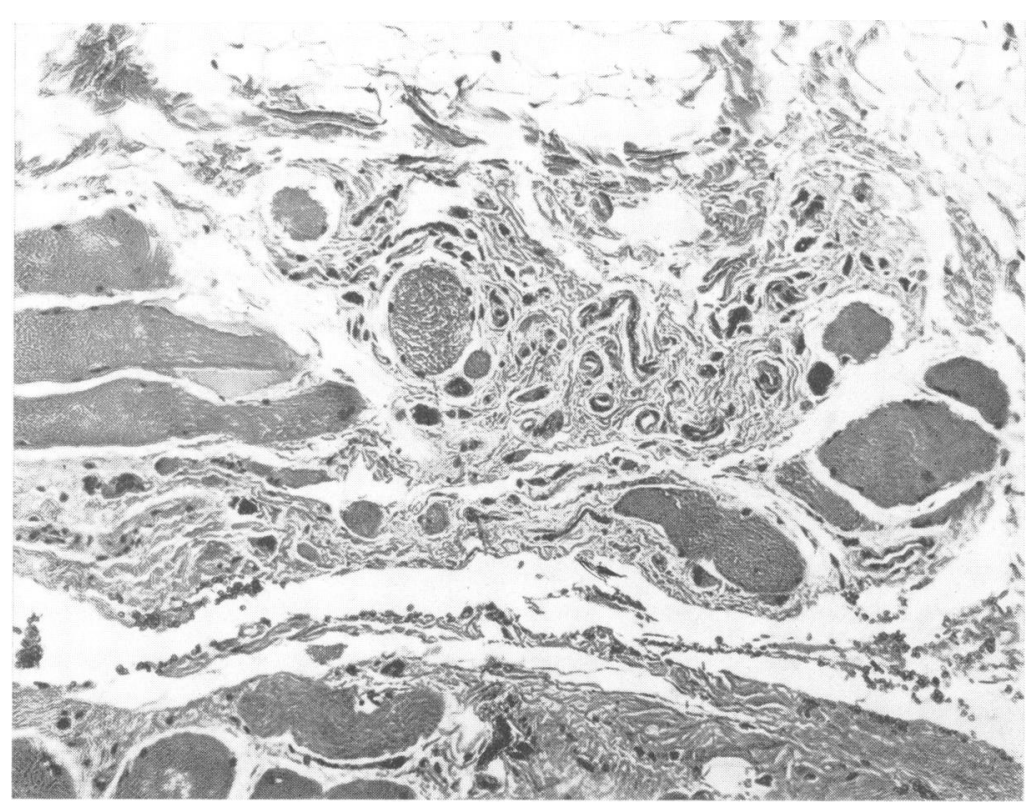

FIG. 5. Left quadriceps

femoris muscle showing denervation, atrophy, and myopathic changes. Weigert's haematoxylin and eosin, $\times 110$.

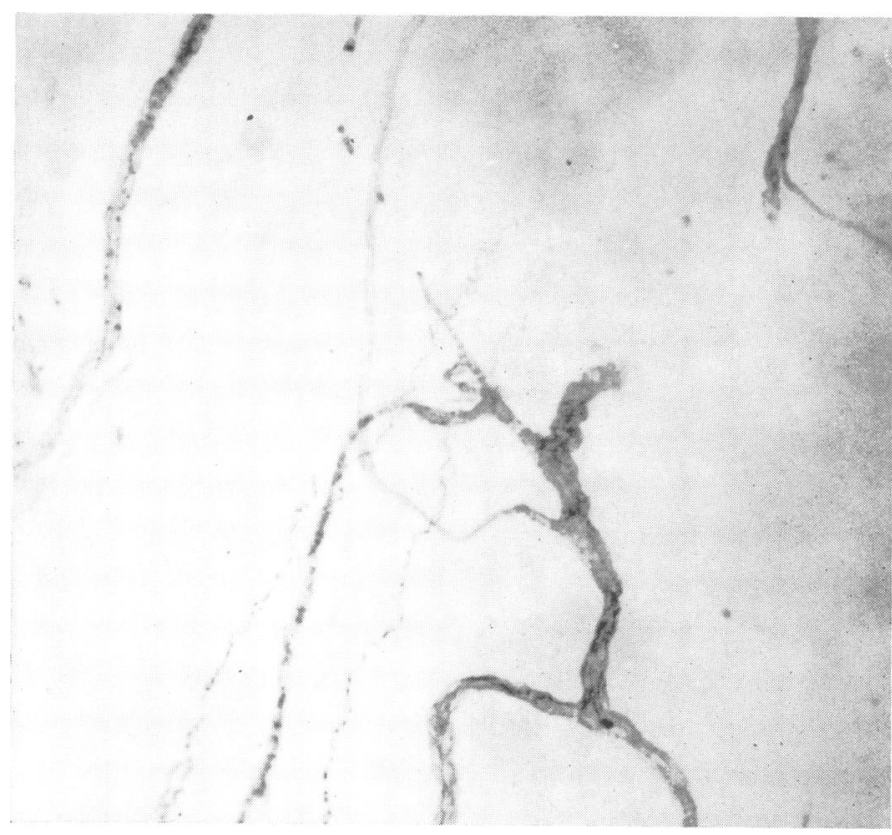

FIG. 6. Left quadriceps femoris muscle. Finely branched axons pursuing a tortuous course amongst abnormal muscle fibres. Squash preparation. Methylene blue, $\times 40$. 


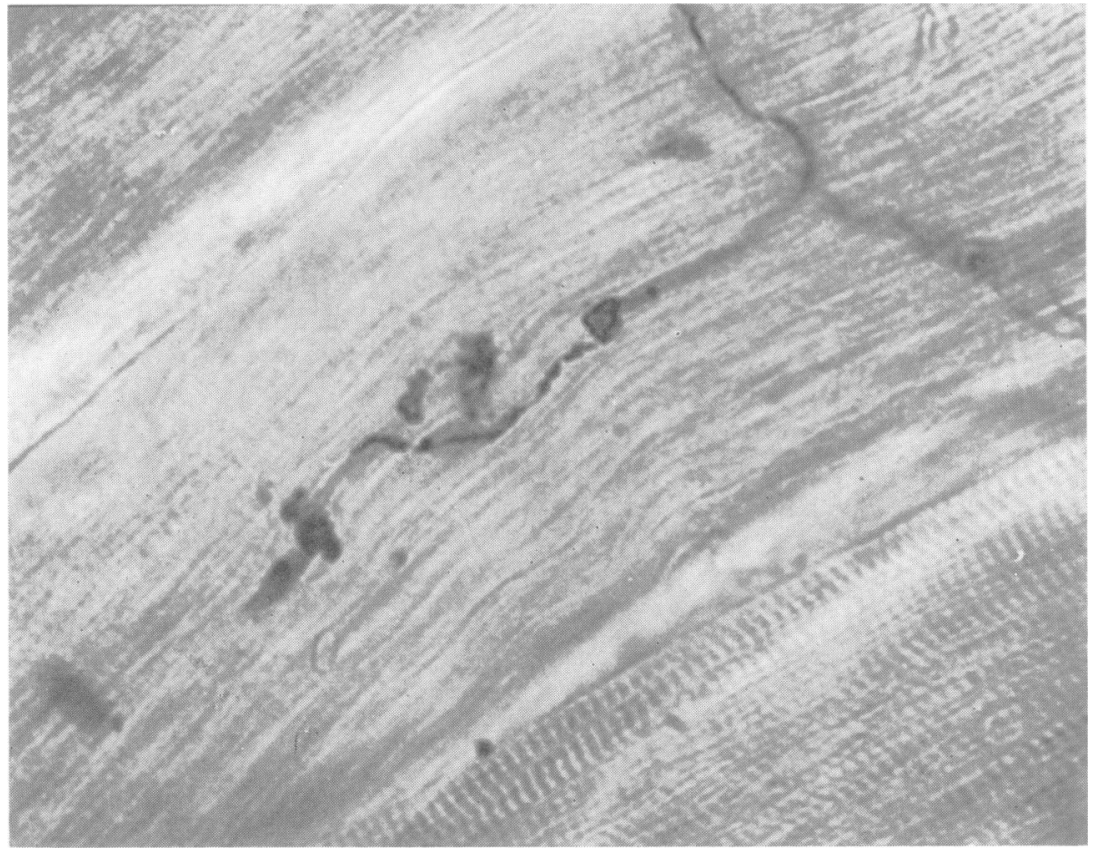

FIG. 7. Left quadriceps femoris muscle. An abnormal motor endplate. Terminal arborization can be seen on a single muscle fibre. Squash preparation Methylene blue, $\times 160$.

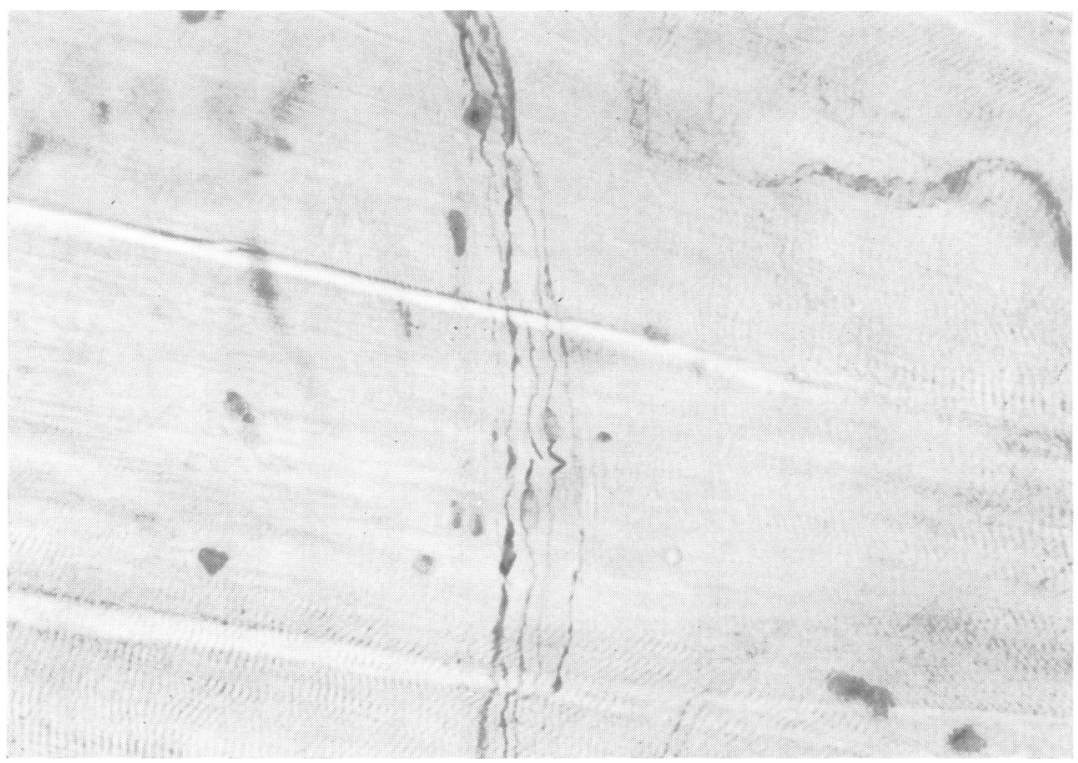

FIG. 8. Left quadriceps femoris muscle. Large oval-shaped swellings can be seen arising from axons in an intramuscular nerve bundle. Squash preparation Methylene blue, $\times 100$. 
were normal. The standard 12 lead electrocardiograph was normal. Electromyography showed no abnormalities in the upper limbs. There were positive sharp waves and a reduced interference pattern in the right tibialis anterior muscle. There were multiple discrete units and a reduced interference pattern in the right extensor digitorum brevis and in the left tibialis anterior muscles. The conduction velocity in the right lateral popliteal nerve was $37.8 \mathrm{~m} / \mathrm{s}$. These findings suggested a chronic partial denervation due to a lesion at nerve root or spinal cord level. A biopsy for histological studies was obtained from the left quadriceps femoris muscle.

\section{CASE 4}

The proband's paternal grandfather J.M. (2-9) had died from pulmonary tuberculosis at the age of 59 years. His son remembered that his father's legs were severely wasted. He had walked with a waddling gait using two walking sticks and had worn highheeled boots to compensate for a deformity of his feet.

The patient's other sister D.M. (4-10) was a perfectly healthy schoolgirl aged 13 years.

The family pedigree is set out in Fig. 4. All surviving members of the family, 14 in number, have been examined by one of us, and no abnormalities were found. There is no known consanguinity. There are no other affected individuals as far as the senior members of the family can recall.

\section{METHODS}

The specimen of muscle from case 3 was carefully excised under general anaesthesia. A thin longitudinal strip was injected immediately after excision with $0.015 \%$ methylene blue solution (Boots Pure Drug Co. Ltd.,) using a fine hypodermic needle. The injected strip of muscle was oxygenated for one hour and then placed in freshly filtered ice cold $8 \%$ ammonium/molybdate solution for 24 hours. After fixation the specimen was rinsed thoroughly in distilled water and squash preparations were made by the method of Dastur (1956). They were then dehydrated in absolute alcohol, cleared in xylol, and mounted in Xam. The remainder of the biopsy specimen was fixed in $10 \%$ formol/calcium. Paraffin sections were stained with Weigert's haematoxylineosin, phosphotungstic acid, haematoxylin periodic acid-Schiff-haematoxylin, Anderson's Victoria Blue, Van Gieson's, and Masson's trichrome stains.

\section{RESULTS}

Conventional light microscopy of the muscle biopsy specimen from J.M. (3-8) revealed a mixed pattern of muscle fibres of normal diameter, of fibres greater than $150 \mu$ in diameter and of fibres less than $10 \mu$ in diameter (Fig. 5).

The atrophied fibres showed clumping and basophilia of their subsarcolemmal nuclei and were often arranged in small groups. More than $3 \%$ of the normal and enlarged muscle fibres showed internally situated nuclei. All muscle fibres examined showed preservation of their cross striations. There was an increase in the amount of fibroelastic and fatty tissue separating isolated normal muscle fibres and groups of normal muscle fibres. Muscle glycogen was detected in apparently normal amounts and was distributed diffusely along the I bands, particularly in type 2 fibres. On the basis of staining for glycogen the atrophied muscle fibres appeared to consist of approximately equal numbers of type 1 and of type 2 fibres. Two muscle spindles were identified. These were within normal limits. Perimysial arterioles and endomysial capillaries showed no abnormalities. No abnormal cellular infiltrations could be seen either around the blood vessels or among the muscle fibres themselves. In vitally stained methylene blue preparations several axons bearing large varicosities along their entire length were seen pursuing a tortuous course amongst the abnormal muscle fibres (Fig. 6). There was a marked degree of sprouting from subterminal axons, and occasional large bizarre shaped terminal arborizations were seen (Fig. 7). Large spherical swellings were also noted along the course of several of the intramuscular and subterminal axons (Fig. 8).

\section{DISCUSSION}

The clinical features of this disorder are severe weakness and wasting of the muscles of the feet, legs, and thighs, with extensor plantar responses. There is no ataxia, and no abnormalities in the cranial nerves, upper limbs, or the trunk. There is no disturbance of sensation. The clinical picture is fairly uniform in all the cases. The only points of difference are that in S.M. (4-9) there is complete areflexia and in J.M. (4-8) there is marked spasticity of the lower limbs.

The disease appears to be inherited as an autosomal dominant character. It differs from the majority of the hereditary neuropathies firstly in being present at birth and showing no tendency 
to progress thereafter. This is in marked contrast with the slow and continuing deterioration seen, for example, in Friedreich's ataxia, limb girdle muscular dystrophy, or dystrophia myotonica. Secondly, three individuals have shown a marked degree of arthrogryposis multiplex congenita. It is well known that muscle biopsies from cases of arthrogryposis multiplex congenita show neuropathic and myopathic features. Smith et al. (1963) found electromyographic evidence of denervation in 17 patients with arthrogryposis multiplex congenita and concluded that the lesion was in the anterior horn cells of the spinal cord. In our cases areflexia was found in S.M. (4-9) and the ankle jerks were depressed in J.M. (3-8). This suggests a neuropathic process. On the other hand, in J.M. (4-8) there was muscle wasting in the thighs with preservation of the knee jerks, suggesting a myopathic process. Unequivocal evidence of a neuropathic process in J.M. (3-8) was shown by electromyographic studies.

Our cases most closely resemble those described by Symonds and Shaw (1926) in which there was weakness of the distal muscles of the upper and lower limbs and equivocal plantar responses. Wasting of the muscles was, however, slight, in marked contrast with our own cases. Some of their cases presented at birth, but in others the disease first appeared in the first, second, or third decade. Rombold and Riley (1926) described a syndrome in three generations of one family which consisted of pes cavus, diminished ankle jerks, and extensor plantar reflexes. In the first generation affected the disease appeared at the age of 17 years, but in the third generation it appeared in two individuals aged 4 and 5 years respectively. It differs from our cases in the complete absence of muscle wasting and in the age of presentation. Kaeser (1965) described 12 cases in one family of 'a slowly progressive spinal muscular atrophy'. This disease began in the distal muscles of the limbs and spread proximally. In later generations it involved the muscles of the neck, shoulder, face, palate, and pharynx. The plantar responses were flexor. This syndrome clearly differs from the cases presented in this paper.

It can be seen that our cases resemble some of the previously listed syndromes but are clearly not identical with any of them. In particular none of the other syndromes regularly presented at birth or showed the features of arthrogryposis multiplex congenita. Our cases seem to be definitely non-progressive. We think that the syndrome merits recognition as a distinct clinical entity.

The overall clinical picture is that of a neuropathy. In one case (case 3) in which the reflexes were preserved one might argue that a distal myopathic process was present. In this case there was definite reduction of the ankle jerks and electromyographic study demonstrated clear evidence of a neuropathic process only. However, in the muscle biopsy from the same case there were findings suggesting a combined neurogenic atrophy and a myopathy. There are reports of similar findings in the literature. Haase and Shy (1960) examined muscle biopsies from 17 cases of peroneal muscular atrophy and their findings were as follows: normal appearances in two, neurogenic lesions only in three, myopathic lesions only in two, and the remaining 100 biopsies showed the combined features of neurod $\frac{\omega}{\sigma}$ pathic and myopathic lesions. Lucas anf Forster (1962) made similar observations.

We feel that the disorder is an inherited neus ropathy. The presence of pyramidal tract sign suggests that the underlying abnormality probably a biochemical one-is shared by some upper motor neurones as swell as by spinal motor neurones. We would classify it as a spinal muscular atrophy. It has been suggested that in longstanding chronic neuropathies myopathic changes may appear in voluntary muscle. This might explain the pathological findings in this case. The reason for the appearance of the secondary myopathic changes in unknown.

We wish to thank Professor W. B. Matthews for permission to publish a report of his patients, and for his help and criticism in the preparation of this paper.

\section{REFERENCES}

Dastur, D. K. (1956). The motor unit in leprous neuritis. A clinico-pathological study. Neurology (Bombay), 4, 1-27.

Haase, G. R., and Shy, G. M. (1960). Pathological changes in No muscle biopsies from patients with peroneal muscular atrophy. Brain, 83, 631-637.

Kaeser, H. E. (1965). Scapuloperoneal muscular atrophy. Brain, 88, 407-418. 
Lucas, G. J., and Forster, F. M. (1962). Charcot-Marie-Tooth disease with associated myopathy. A report of a family. Neurology (Minneap.), 12, 629-636.

Rombold, C. R., and Riley, H. A. (1926). The abortive type of Friedreich's disease. Archives of Neurology and Psychiatry, 16, 301-312.

Roth, M. (1948). On a possible relationship between hereditary ataxia and peroneal muscular atrophy; with a critical review of the problems of 'intermediate forms' in the degenerative disorders of the central nervous system. Brain, 71, 416-433.

Smith, E. M., Bender, L. F., and Stover, C. N., Arbor, A., (1963). Lower motor neuron deficit in arthrogryposis. An EMG study. Archives of Neurology (Chic.), 8, 97-100.

Symonds, C. P., and Shaw, M. E. (1926). Familial claw-foot with absent tendon-jerks: a 'forme fruste' of the MarieCharcot-Tooth disease. Brain, 49, 387-403. 